21 and 27 years old (all pregnancies went to term). She became pregnant again at the age of 31 and since she was treated with numerous drugs with possible teratogenic effect, abortion was recommended. The early termination of this pregnancy was successfully achieved by aspiration alone during the tenth week. Abortion was immediately followed by a manic episode with psychotic features which resolved with conventional treatment. Mrs A. experienced a fifth pregnancy at the age of 33 (to term, Caesarean section) which was associated with a manic episode with psychotic features. At the age of 35 she experienced a sixth pregnancy which stopped spontaneously at the tenth week. This spontaneous abortion was followed by a manic state. At the age of 36 she experienced a seventh pregnancy (to term) which was followed by a manic episode with psychotic features. Delivery was followed by a marked decrease in peripheral oestrogen. Peripheral oestrogen crosses the blood-brain barrier and modulates various systems of neurotransmission, especially dopaminergic transmission (Fink et al, 1996), and the oestrogen decrease has been hypothesised as being a precipitating factor of puerperal psychosis in predisposed individuals (Deuchar \& Brockington, 1998).

The occurrence of both puerperal psychotic mania and abortion-associated psychotic mania in the same person suggests an individual predisposition, and a single aetiological mechanism can be hypothesised: abortion (spontaneous or induced) during the first trimester is followed by a marked decrease in oestrogen levels (Blazar et al, 1980) and the termination of all seven pregnancies was simultaneously associated with both the occurrence of acute psychotic mania and a decrease in oestrogen levels consistent with a modification of the brain oestrogen environment. This suggests that the oestrogen withdrawal hypothesis may be relevant in psychoses occurring after pregnancies that are not going to term.

Blazar, A. S., Harlin, J., Zaidi, A. A., et ol (1980) Differences in hormonal patterns during the first postabortion menstrual cycle after two techniques of termination of pregnancy. Fertility and Sterility, 33, 493-500.

Brewer, C. (1977) Incidence of post-abortion psychosis: a prospective study. British Medical Journal, I, 476-477.

Deuchar, N. \& Brockington, I. (1998) Puerperal and menstrual psychoses: the proposal of a unitary aetiological hypothesis. Journal of Psychosomatics Obstetrics \& Gynecology, 19, 104-110.

Fink, G., Sumner, B. E., Rosie, R., et al (1996)

Estrogen control of central neurotransmission: effect on mood, mental state, and memory. Cellular \& Molecular Neurobiology, 16, 325-344.

V. Mahe, F. Montagnon, J. Nartowski, A.

Dumane Service de Psychiatrie, Hôpital Général de Meaux, 6-8 rue Saint-Francis, 77100 Meaux, France

\section{Mirtazapine withdrawal causing hypomania}

Sir: Although a number of studies have reported the development of hypomania following discontinuation of antidepressant therapy (e.g. Mirin et al, 1981; Hartmann, 1990; Landry \& Roy, 1997), this has not yet been reported for the relatively new noradrenergic and specific serotonergic antidepressant, mirtazapine.

A 65-year-old woman was commenced on mirtazapine $30 \mathrm{mg}$ nocte owing to symptoms of depressed mood with diurnal variation (worse in the morning), intermittent suicidal ideation, disrupted sleep pattern, poor concentration, lack of motivation and general anhedonia. Although she derived little benefit from the medication, she remained on it for a period of five weeks at which point of her own accord, she decided to discontinue it abruptly at a time when her suicidal ideation was particularly marked. Within two days of stopping the drug, she felt dramatically better and became quite elated and mildly disinhibited, with pressure of speech, increased energy levels and a reduced need for sleep. She adopted a youthful style of dress and demeanour and offered one of the authors small gifts. At no point during this time, however, did she develop flight of ideas or any psychotic symptoms. She continues to be mildly hypomanic six weeks after drug withdrawal.

This woman had a long history of recurrent anxiety and depressive episodes. She had previously suffered a mild degree of hypomania following commencement of paroxetine in July 1998 but there is no other history of hypomania. Prior to commencing mirtazapine, she had been treated unsuccessfully with sertraline in doses up to $100 \mathrm{mg}$ daily (she was unwilling to increase the dose further because of side-effects and lack of efficacy). Although hypomania has been noted to have occurred following mirtazapine augmentation of sertraline (Soutullo et al, 1998), the symptoms of hypomania only occurred in this case immediately after discontinuation of the mirtazapine and the two drugs were never given concurrently.

Mechanisms proposed to explain this withdrawal phenomenon previously noted with other classes of antidepressant include 'cholinergic overdrive' (Dilsaver \& Greden, 1984) and noradrenergic hyperactivity (Charney et al, 1982), although the true reason for its occurrence remains unclear. This further report of the same phenomenon with one of the newer classes of antidepressant serves to highlight the need for further research in this area.

Chamey D. S., Heninger, G. R., Sternbers, D. E., ot al (1982) Abrupt discontinuation of tricyclic antidepressant drugs: evidence for noradrenergic hyperactivity. British journal of Psychiatry. 141, 377-386.

Dilsaver, S. C. \& Groden, J. F. (1984) Antidepressant withdrawal-induced activation (hypomania and mania): mechanism and theoretical significance. Brain Reseorch, 319. 29-48.

Hartmann, P. M. (1990) Mania or hypomania after withdrawal from antidepressants. Journal of Fomily Proctice, 30. 47I-472.

Landry, P. \& Roy, L. (1997) Withdrawal hypomania associated with paroxetine. Journal of Clinical Psychopharmocology. 17, 60-61.

Mirin, S. M., Schatzberz, A. F. \& Creasey, D. E. (1981) Hypomania and mania after withdrawal of tricyclic antidepressants. American journal of Psychiotry. 138, 87-89.

Soutullo, C. A., McElroy, S. L., Keck, P. E., Jr (1998) Hypomania associated with mirtazapine augmentation of sertraline. Journal of Clinical Psychiotry, 59. 320.

C. MacCall, J. Callender Block A. Clerkseat, Royal Cornhill Hospital, Cornhill Road, Aberdeen AB25 2ZH

\section{Drug therapy in treatment-resistant depression}

Sir: We commend the study by Poirier \& Boyer (1999), comparing the efficacy of venlafaxine and paroxetine in people with treatment-resistant depression as this is a difficult sub-population in which to conduct research. However, we were dismayed that there was no discussion as to why venlafaxine should be superior to paroxetine, a finding that is indeed supported by our clinical experience in managing people with treatment-resistant depression.

The majority of our referrals with treatment-resistant depression have usually been prescribed adequate doses of tricyclic antidepressants, selective serotonin reuptake inhibitors and monoamine oxidase inhibitors during the course of their illness. It is our practice to use augmentation strategies, 